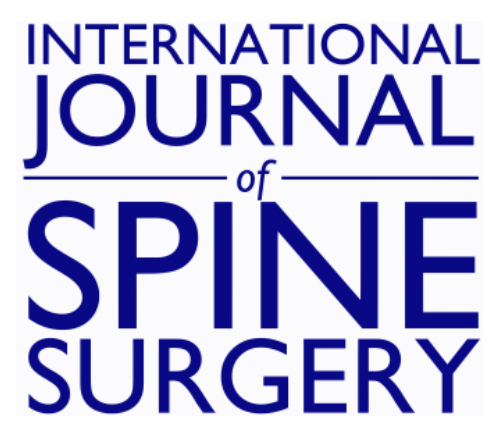

\title{
Association of Preoperative Hemoglobin A1c and Body Mass Index with Wound Infection Rate in Spinal Surgery
}

Miner N. Ross, Sudarshan Iyer, Kenneth R. Gundle and Donald A. Ross

Int J Spine Surg 2021, 15 (4) 811-817

doi: https://doi.org/10.14444/8104

http://ijssurgery.com/content/15/4/811

This information is current as of April 25, 2023.

Email Alerts Receive free email-alerts when new articles cite this article. Sign up at:

http://ijssurgery.com/alerts

The International Journal of Spine Surgery

2397 Waterbury Circle, Suite 1,

Aurora, IL 60504, Phone: +1-630-375-1432 


\title{
Association of Preoperative Hemoglobin A1c and Body Mass Index with Wound Infection Rate in Spinal Surgery
}

\author{
MINER N. ROSS, MD, MPH, ${ }^{1}$ SUDARSHAN IYER, ${ }^{1}$ KENNETH R. GUNDLE, MD, ${ }^{2,3}$ \\ DONALD A. ROSS, MD ${ }^{1,2}$ \\ ${ }^{1}$ Department of Neurological Surgery, Oregon Health \& Science University, Portland, Oregon, ${ }^{2}$ Operative Care Division, Portland Veterans Affairs Medical \\ Center, Portland, Oregon, ${ }^{3}$ Department of Orthopaedics and Rehabilitation, Oregon Health \& Science University, Portland, Oregon
}

\begin{abstract}
Background: The deleterious effect of diabetes mellitus on surgical outcomes is well documented for joint replacement surgery. We analyzed the large national US Department of Veterans Affairs (VA) database for patients who had undergone elective spinal surgery.

Methods: We retrospectively searched the VA database and identified 174520 spine cases.

Results: There were 7766 (4.5\%) wound infections and 49271 (28\%) had hemoglobin A1c (HbA1c) testing (range: 3.0-17.8) prior to surgery. In the preoperative HbAlc-checked group, there were 2941 (6.0\% of 49271$)$ infections and in the without-preoperative HbAlc group, there were 4825 (3.9\% of 125249$)$ infections. The distribution of infections was significantly different $\left(\chi^{2}=372.577, P<.0001\right)$ and suggests a $2.12 \%$ increase in the absolute risk of infection based on the presence of preoperative HbA1c testing, regardless of the result. Logistic regression revealed a preoperative HbA1c test was associated with an odds ratio of 1.435 for infection (confidence interval 1.367-1.505, $P<$ .0001). In a separate model based on HbAlc levels, we found that HbAlc is a significant predictor of infection with an odds ratio of 1.042 (confidence interval $1.017-1.068, P=.0009$ ) for each $1 \%$ increase in the test result. This analysis differs from using a strict cutoff value of HbAlc of $6.5 \%$. Similar testing for body mass index and age yielded an odds ratio of 1.027 for each increase of $1 \mathrm{~kg} / \mathrm{m}^{2}$ and an odds ratio of 1.009 for each 1 -year increase in age respectively.

Conclusions: Hemoglobin Alc testing, $\mathrm{HgAlc}$ value, body mass index, and age all contribute to the risk of wound infection after elective spine surgery in a large national VA population. These data can be used to estimate surgical risks and to aid in patient counseling about proposed spine surgery.
\end{abstract}

Level of Evidence: 4.

Other \& Special Categories

Keywords: veterans, spine, BMI, hemoglobin A1c, wound infection

\section{INTRODUCTION}

In recent years, diabetes mellitus has emerged as a common and significant comorbidity in the United States. The latest compiled statistics from the Centers for Disease Control and Prevention estimate that 30.2 million US adults, $(12.2 \%$ of the adult population) have diabetes, including 7.2 million who are undiagnosed. ${ }^{1} \mathrm{~A}$ further 84.1 million adults $(33.9 \%)$ had prediabetes in 2015 , including $48.3 \%$ of all adults aged 65 or older. ${ }^{2}$ Notably, evidence also shows that the prevalence of diabetic patients undergoing spinal surgery is on the increase. ${ }^{3,4}$

The deleterious effect of uncontrolled diabetes on surgical outcomes is well documented in the literature, ${ }^{5}$ particularly with respect to joint replacement surgery $^{6-11}$ and peripheral nerve surgery. ${ }^{12}$
Less well described is the relationship between diabetes and outcomes in spinal surgery. ${ }^{13,14}$ In this study, we undertake a national retrospective database review to model any risk relationship between preoperative obesity and glycemic control (as approximated by body mass index [BMI] and hemoglobin A1c [HbAlc], respectively) and postsurgical wound infection in patients undergoing surgery for degenerative spine conditions.

\section{METHODS}

With the consent of the Institutional Review Board, a request was made of the US Department of Veterans Affairs (VA) corporate data warehouse for access to the national VA database in Austin, Texas, using the VA Informatics and Computing Infrastructure. A request was made for information on 
all VA patients undergoing elective spinal surgery for degenerative spine conditions from years 2007 to 2016. Spine surgeries were included based on common procedure terminology (CPT) codes. The operations included laminectomies (CPT 63005, 63017, 63042, 63045, 63047), discectomies (63030), foraminotomies $(63045,63047,63048)$, anterior cervical discectomies and fusions $(63075,22551$, 22845), cervical arthroplasties (22856), cervical laminoplasties (63050), posterior cervical fusions $(22590,22595,22600,22614)$, anterior lumbar fusions (22558, 22585, 22845, 22851), and posterior lumbar fusions (22612, 22630, 22840, 22842, 22843). Surgeries for trauma, infection, or neoplasms were excluded. Information was extracted including patient age, date of surgery, weight and height of the patients as recorded in the 3 months prior to surgery, HbAlc levels measured in the 3 months prior to surgery, and complications of surgery. Complications were identified by ICD codes 996.66 (infection due to internal implant or prosthesis), 996.67 (infection due to other orthopedic device, implant, or graft), 998.59 (other postoperative infection), 998.83 (nonhealing surgical wound), 998.89 (other complications of procedures), and T81.4XXA (infection following a procedure), which were recorded in the 6 months following the index procedure. These were combined into a single primary outcome: presence of an infectious complication.

Statistical analysis and modeling were performed using the SAS 9.2 statistical package (2008, SAS Institute, Cary, NC). Based upon the dichotomous nature of the outcome variable infection and the continuous nature of predictor variables of $\mathrm{HbAlc}$, age, and BMI, we selected logistical regression analysis a priori. STROBE guidelines for reporting observational studies ${ }^{15}$ were used in the design and reporting of this study. Data are presented as mean $\pm \mathrm{SD}$.

\section{RESULTS}

The initial query resulted in 430000 individual CPT codes. Following removal of redundant entries with multiple CPT codes for the same procedure, and procedures not meeting criteria, there were 191320 unique procedures. Of these patients, 175895 had sufficiently recorded height and weight to calculate BMI prior to surgery. We elected to exclude patients with BMI $<15$ and $\mathrm{BMI}>50$ to prevent the inclusion of significant
Table 1. Patient characteristics.

\begin{tabular}{lr}
\hline Characteristic & n (\%) \\
\hline Sex & \\
$\quad$ Male & $163473(92.94)$ \\
Female & $12422(7.06)$ \\
Race & $126110(71.70)$ \\
White & $30128(17.13)$ \\
Black & $1821(1.04)$ \\
Native American & $1711(0.97)$ \\
Hawaiian or Pacific Islander & $723(0.41)$ \\
Asian & $15402(8.76)$ \\
Unknown/declined to state & $7223(4.11)$ \\
Ethnicity & $168672(95.89)$ \\
Hispanic/Latino & \\
Non-Hispanic/Latino & \\
\hline
\end{tabular}

outliers and decimal point errors in the model. The decision to exclude BMI outliers was based on a preliminary analysis showing significant positive skew of the distribution of BMI that corrected after application of these boundaries. This ultimately excluded $0.78 \%$ of cases and resulted in a final population of 174520 patient cases for analysis. A summary of patient characteristics is provided in Table 1. Using the text descriptors of the procedures performed, we tabulated the types of operations these patients received and grouped them by approach (anterior, posterior, lateral, or unspecified), by spinal segment (cervical, thoracic, lumbar, multiple, or unspecified), and by whether the surgery involved decompression alone or the placement of hardware; a summary is provided in Table 2. Many of the surgeries could not be precisely characterized due to the nonspecific text titles of the procedures supplied.

The mean age at surgery was 59 years $( \pm 13$ years). In the analyzed population, 49271 patients $(28 \%)$ had their HbA1c tested prior to surgery. Values of HbA1c in this group ranged from 3.0 to 17.8. We suspected that many patients in this

Table 2. Spine surgeries.

\begin{tabular}{lc}
\hline Surgical Details & n (\%) \\
\hline Approach & \\
Anterior & $20583(11.79)$ \\
Posterior & $56269(32.24)$ \\
Lateral & $126(0.07)$ \\
Unspecified & $97542(55.89)$ \\
Segment & \\
Cervical & $11905(6.82)$ \\
Thoracic & $1059(0.61)$ \\
Lumbar & $34408(19.72)$ \\
Multiple or unspecified & $127148(72.86)$ \\
Type & $65042(37.27)$ \\
Decompression alone & $48828(27.98)$ \\
Fusion and/or instrumentation & $6650(34.75)$ \\
Unspecified &
\end{tabular}


Table 3. Predictors of infection.

\begin{tabular}{lccr}
\hline Models & $\begin{array}{c}\text { Odds } \\
\text { Ratio }\end{array}$ & $\begin{array}{c}\text { Confidence } \\
\text { Interval }\end{array}$ & $\boldsymbol{P}$ Value \\
\hline Model 1: continuous HbA1c & & \\
Age & 1.009 & $1.006-1.012$ & $<.0001$ \\
BMI & 1.027 & $1.020-1.034$ & $<.0001$ \\
HbA1c & 1.042 & $1.017-1.068$ & .0009 \\
Model 2: presence vs absence of HbA1c & & \\
Age & 1.015 & $1.013-1.017$ & $<.0001$ \\
BMI & 1.030 & $1.025-1.034$ & $<.0001$ \\
HbAlc presence & 1.435 & $1.367-1.505$ & $<.0001$ \\
\hline
\end{tabular}

Abbreviations: BMI, body mass index; HbAlc, hemoglobin Alc.

population were being screened for preclinical diabetes by HbAlc sampling; therefore, we examined differences in age and BMI, stratified by whether HbAlc had been tested. We found that BMI was significantly greater in the tested group (30 vs 28 in the untested group, $P<.0001)$. Likewise, the patients who had HbAlc testing were significantly older than those who were untested (62 vs 58 years, $P<.0001)$. Given these findings, we elected to include age as well as BMI in our regression model.

Of 174520 patients, there were 7766 postoperative infections $(4.5 \%)$. In the group that had a preoperative HbA1c checked, there were 2941 such infections $(6.0 \%$ of 49271$)$, while in the group without a preoperative $\mathrm{HbA1c}$, there were 4825 infections $(3.9 \%$ of 125249$)$. The distribution of infections in these 2 groups was significantly different from their expected proportions $\left(\chi^{2}=\right.$ $372.577, P<.0001)$ and suggests a $2.12 \%$ increase in the absolute risk of infection based on the mere presence of preoperative $\mathrm{HbA} 1 \mathrm{c}$ testing, regardless of the result.

Due to the nature of the coding of the procedures in our database, extraction of approach and spinal level is available for a minority of the patients, and many of these have one or the other only specified. This is a known drawback of large database analysis based upon the coding acumen and heterogeneous coding practices of many institutions. A subset analysis of a sample of the first 4000 patients in the database yielded the following: Among the surgeries with resulting infections, $34.6 \%$ were cervical, $1 \%$ were thoracic, $35.6 \%$ were lumbar (59.5\% fusions), $25 \%$ were at an unknown spinal level, and $3.8 \%$ involved a stimulator or pump device; $68.3 \%$ of infections involved a fusion or an implanted stimulator or pump. Infected procedures were $9.6 \%$ anterior without fusion, $22.1 \%$ posterior without fusion, $38.5 \%$ posterior with fusion,
$19.2 \%$ anterior with fusion, and $6.7 \%$ with fusion of unknown approach. This is consistent with published data of wound infections in spinal surgery. ${ }^{13,14}$

Infection rates were also reviewed by race/ ethnicity. In the Veterans Administration, Hispanic or Latino is an ethnicity attached to either White or Black races, so patients are counted in both groups. Therefore, the total numbers differ from the total population as noted above. The infection rates of the entire population with $\mathrm{HbA} 1$ values were $4.7 \%$ among Whites, 3.5\% among Blacks, $4.0 \%$ among Hispanics, $4.3 \%$ among Hawaiian and other Pacific Islanders, 2.5\% among Asians, and $4.7 \%$ among Native Americans and Alaska Natives. Black veterans constituted $17.13 \%$ of our population, but only $14.5 \%$ of the infections; therefore, there did not seem to be a predisposition to infection among Black veterans (see "Discussion," below).

Logistic regression models were constructed using the following as possible predictors for infectious complications: age at surgery, BMI, HbAlc level, and the dichotomized presence vs absence of HbAlc testing in the patient's chart. We used both $\mathrm{HbAlc}$ as a continuous variable and as a dichotomized variable (tested for or not tested for) in separate analyses. Odds ratios and $P$ values for these models are summarized in Table 3. Due to high covariance, $\mathrm{HbA} 1 \mathrm{c}$ level and the categorical variable for the presence of preoperative HbA1c testing were not included in the same regression model. In its own model, the presence of a preoperative HbAlc test was associated with an odds ratio of 1.435 for infection (confidence interval $1.367-1.505, P<.0001$ ). In a separate model based on HbAlc levels, we found that $\mathrm{HbA1c}$ is a significant predictor of infection with an odds ratio of 1.042 (confidence interval 1.017$1.068, P=.0009$ ) for each $1 \%$ increase in the test result. Similar testing for BMI and age yielded an odds ratio of 1.027 for each increase of $1 \mathrm{~kg} / \mathrm{m}^{2}$ and an odds ratio of 1.009 for each 1-year increase in age.

\section{DISCUSSION}

Diabetes mellitus is a known risk factor for adverse surgical outcomes. ${ }^{5,7,8}$ In a study of 7565 elective and emergency surgeries requiring at least 1 night of hospitalization (including 724 neurosurgical and 1000 orthopedic procedures), Yong et $\mathrm{al}^{5}$ found 
that diabetes mellitus, defined as $\mathrm{HbA} 1 \mathrm{c} \geq 6.5 \%$, was associated with increased 6-month mortality, major complications, intensive care unit admission, mechanical ventilation, and hospital length of stay, even after adjustment for age, Charlson comorbidity index, glomerular filtration rate, and length of surgery. In total hip arthroplasty, elevated HbAlc levels have been correlated with increased cost of care. ${ }^{7}$ In reviewing the current evidence about diabetes mellitus and risk of arthroplasty, Lopez et $\mathrm{al}^{8}$ concluded that while diabetes increases the risk for postoperative complications, there is no evidence to determine if this risk is related to glycemic control as measured by HbAlc.

Prior findings in the spinal surgery literature have been mixed regarding the contribution of diabetes mellitus to the risk of postoperative wound infection and the role of $\mathrm{HbAlc}$ in predicting this risk, though there is a broad consensus that glycemic control must play some role. In a retrospective review of 165 patients, Takahashi et $\mathrm{al}^{16}$ found that while factors related to diabetes predict poor recovery from lumbar spinal surgery for criteria such as improvement in back pain or lower extremity numbness, there was no significant difference in the incidence of infection or reoperation between their diabetic and nondiabetic patients. However, their study may have been underpowered for detecting this endpoint.

A large database analysis of 423050 diabetic patients undergoing degenerative lumbar surgery by Guzman et $\mathrm{al}^{3}$ in 2014 found increased odds of medical complications and postoperative infection in uncontrolled diabetics relative to controlled diabetics. Uncontrolled and controlled diabetes were defined by diagnosis codes and not by HbA1c. The odds ratio for postoperative infection of any type was 2.61 for uncontrolled diabetes and 1.36 for controlled diabetes. The same group also looked at cervical spinal surgery, reporting that patients with uncontrolled diabetes had higher risks for postoperative infection (odds ratio 4.90), pulmonary embolism, inpatient mortality, and length of stay than patients with either controlled diabetes (for infection: odds ratio 1.91 ) or no diabetes. ${ }^{4}$ However, the same group in a subsequent study detected no significant difference in the odds of infection based on diabetic status after adult scoliosis surgery, ${ }^{17}$ though they did observe that diabetic status is a predictive factor of other known postoperative complications such as acute renal failure and hemorrhage.

A more recent study ${ }^{18}$ examined a cohort of 523 diabetic patients who underwent posterior lumbar surgery with a wound infection rate of $7.1 \%$. They found that HbAlc $\geq 7.50$ increased the odds of a wound infection significantly, but did not find a correlation between age or BMI and wound infection risk. Notably, their analysis only included HbAlc dichotomized at the level of $7.5 \%$, which may have affected these conclusions.

Other studies have found correlation of diabetes with postoperative spinal wound infection, but did not document the criteria for the diagnosis of diabetes, for inclusion in the control group, or for the degree of glycemic control ${ }^{13}$ or used medical record criteria or nonrandom, nonfasting serum glucose levels during hospitalization or at a preoperative clinic visit for some patients in the study. ${ }^{14}$

Our study examines a large cohort and establishes a dose-response relationship between BMI or HbAlc and the odds of infection. Given an overall incidence of wound infection of $4.45 \%$ in our study population, it is reasonable under the rare disease assumption to consider the odds ratios generated by our model as approximating the relative risk of infection. The units of HbAlc are percent and the units of BMI are $\mathrm{kg} / \mathrm{m}^{2}$. As such, we can state that in this population, a $1 \%$ increase in $\mathrm{HbAlc}$ is associated with an increase of $4.2 \%$ in relative risk and $0.19 \%$ in absolute risk of postoperative infection. A $1 \mathrm{~kg} / \mathrm{m}^{2}$ increase in BMI in the same population was associated with an increase of $2.7 \%$ in relative risk and $0.12 \%$ in absolute risk of infection. In comparison, a 1-year increase in age was associated with an increase of $0.9 \%$ in relative risk or $0.04 \%$ in absolute risk of infection, which indicates that both BMI and HbAlc are stronger predictors of infection.

The presence of preoperative HbAlc testing, regardless of the resultant $\mathrm{HbAlc}$ level, was associated with a $43.5 \%$ increase in the relative risk of infection, corresponding to a $2.12 \%$ increase in the absolute risk of infection in the study population. As preoperative diabetes screening is uncommon in elective spinal surgery at our institution in patients not documented to have diabetes mellitus, we assume that the majority of the HbAlc tests in our study were ordered by primary care providers, either based on a history of 
diabetes mellitus or strong suspicion of an underlying diagnosis. Preoperative $\mathrm{HbAlc}$ testing has become standard practice in other surgical environments. A recent study by Shohat et $\mathrm{al}^{10}$ in 2018 found that $8 \%$ of patients $(123 / 1461)$ undergoing joint arthroplasty were found to have undiagnosed diabetes via preoperative HbAlc measurement, thus suggesting that diabetes screening performed prior to surgical care can provide important information to an operating team. In our experience, preoperative diabetes screening is not standard practice in spinal surgery. As our data suggest that risk of postoperative infection significantly increases with suspicion of diabetes and that the possibility exists of diabetes being undiagnosed before surgical referral, we recommend that preoperative testing of $\mathrm{HbAlc}$ prior to spinal surgery be incorporated in presurgical screening moving forward. Furthermore, as the aforementioned increase in relative risk of infection associated with preoperative $\mathrm{HbA} 1 \mathrm{c}$ testing includes patients with HbA1c levels <6.5 (no diabetes or controlled diabetes), we strongly believe that obesity and other features of metabolic syndrome may also predispose spine surgical patients to infection. Thus, future directions for investigation may include evaluation of broader metabolic syndrome as it contributes to the risk of postoperative spine infection, including risk factors such as hypertension and hyperlipidemia that have not previously been studied in this context.

Attainment of optimal preoperative glycemic control is presumed to reduce the risk of postoperative infection. While the results of our study corroborate this by suggesting that lower $\mathrm{HbAlc}$ levels are associated with reduced risk of infection, this finding is somewhat diminished by the large additional risk associated with the presence of $\mathrm{HbA1c}$ testing preoperatively. Nonetheless, emerging evidence in the evaluation of glycemic control is promising with respect to further clarifying preoperative optimization for diabetic patients. Shohat et $\mathrm{al}^{19}$ in 2017 related an emerging marker, serum fructosamine, to the incidence of periprosthetic joint infection. Fructosamine levels $\geq 292 \mu \mathrm{mol} / \mathrm{L}$ had a significant association with deep wound infection, but $\mathrm{HbA} 1 \mathrm{c}$ level $\geq 7 \%$ did not. Ngaage et $\mathrm{al}^{20}$ provide an excellent review of fructosamine and 2 other serum biomarkers (glycosylated albumin and 1,5-anhydroglucitol) and propose further investigation into their use as predictors of outcomes in spinal surgery. It is possible that similar evaluation of these newer markers of glycemic control will shed light on the exact relationship between diabetes, its management, and the risks associated with spinal surgery.

Infection rates among various ethnicities in our group cannot be attributed strictly to their ethnic origin, as ethnicity may be a surrogate for where the patient lives (such as Pacific Islanders in Hawaii), poverty, or other factors. The dataset we analyzed is overwhelmingly White, so the results of a subgroup analysis by race are not generalizable. Infections after instrumented fusions have been previously reported to be more common than in surgeries without implants ${ }^{3}$ and more likely in posterior fusions than anterior fusions. ${ }^{4}$

We did not review our cohort for cardiopulmonary disease or other confounding medical factors that have been reported to influence readmission rates and complications of spinal surgery. In a study looking at 111892 spine surgery patients in the National Surgical Quality Improvement Project database and using 68 preoperative characteristics, diabetes mellitus did not seem to correlate with readmission or reoperation, although age, African American (Black) race, recent weight loss, chronic steroid use, dialysis, blood transfusion, American Society of Anesthesiologists classification $\geq 3$, contaminated wound, and operative time $>3$ hours did correlate. $^{21}$ We did not find a risk associated with African-American race. In another similar study in 18602 patients undergoing spinal fusion surgery, diabetes mellitus was not shown to correlate with readmission and major perioperative complications $(P=.097) .^{22}$ In 7016 patients undergoing lumbar decompression, diabetes mellitus did correlate in a univariate, but not a multivariate analysis, with unplanned readmission. ${ }^{23}$ In 4793 patients undergoing lumbar decompression without or with fusion, diabetes mellitus was not associated with readmission in a multivariate analysis. ${ }^{24}$

Other study limitations include the retrospective, nonrandomized nature. We cannot verify a diagnosis of diabetes in patients having HbAlc tested preoperatively, but as discussed above, the mere fact that patients were tested suggests either a diagnosis of diabetes or a strong suspicion of the same. Identification of the types of surgeries and the presence of infections relied upon CPT and ICD ontologies. While this is a common methodology in large-scale retrospective database studies, 
there are potential risks of miscoding and incomplete coding that bias our analysis. ${ }^{25}$ The infection rates may therefore represent a low estimate. The biologic plausibility of the associations we describe and similar findings reported in other clinical scenarios bolster the validity of this study. The postoperative infection rate we report is similar to that in other studies, ${ }^{18}$ suggesting that the inclusion of a large number of miscoded infections seems less likely. Finally, it is important to note that the veteran population profiled in this study is overwhelmingly male and Caucasian (White), which reduces the external validity of our findings to some degree. The strengths of this study include the large size of the patient population, the non-self-reported nature of the weight and BMI data, the actual HbA1c values, and the heterogeneous nature of the procedures and surgeons at multiple institutions, which aids in broad applicability.

\section{REFERENCES}

1. Centers for Disease Control and Prevention. 2017. National diabetes statistics report. https://www.cdc.gov/ diabetes/data/statistics/statistics-report.html. Accessed November 1, 2019.

2. Centers for Disease Control and Prevention. 2017. Prevalence of prediabetes. https://www.cdc.gov/diabetes/data/ statistics-report/prevalence.html. Accessed November 1, 2019.

3. Guzman JZ, Iatridis JC, Skovrlj B, et al. Outcomes and complications of diabetes mellitus on patients undergoing degenerative lumbar spine surgery. Spine (Phila Pa 1976). 2014;39(19):1596-1604.

4. Guzman JZ, Skovrlj B, Shin J, et al. The impact of diabetes mellitus on patients undergoing degenerative cervical spine surgery. Spine (Phila Pa 1976). 2014;39(20):1656-1665.

5. Yong PH, Weinberg L, Torkamani N, et al. The presence of diabetes and higher $\mathrm{HbAlc}$ are independently associated with adverse outcomes after surgery. Diabetes Care. 2018;41(6):1172-1179.

6. Cancienne JM, Deasey MJ, Kew ME, Werner BC. The association of perioperative glycemic control with adverse outcomes within 6 months after arthroscopic rotator cuff repair. Arthroscopy. 2019;35(6):1771-1778.

7. Kurowicki J, Rosas S, Khlopas A, et al. Impact of perioperative $\mathrm{HbAlc}$ on reimbursements in diabetes mellitus patients undergoing total hip arthroplasty: a nationwide analysis. J Arthroplasty. 2018;33(7):2038-2042.

8. Lopez LF, Reaven PD, Harman SM. Review: the relationship of hemoglobin A1c to postoperative surgical risk with an emphasis on joint replacement surgery. $J$ Diabetes Complications. 2017;31(12):1710-1718.

9. Shohat N, Foltz C, Restrepo C, Goswami K, Tan T, Parvizi J. Increased postoperative glucose variability is associated with adverse outcomes following orthopaedic surgery. Bone Joint J. 2018;100-B(8):1125-1132.

10. Shohat N, Goswami K, Tarabichi M, Sterbis E, Tan TL,
Parvizi J. All patients should be screened for diabetes before total joint arthroplasty. J Arthroplasty. 2018;33(7):2057-2061.

11. Shohat N, Restrepo C, Allierezaie A, Tarabichi M, Goel $\mathrm{R}$, Parvizi J. Increased postoperative glucose variability is associated with adverse outcomes following total joint arthroplasty. J Bone Joint Surg. 2018;100(13):1110-1117.

12. Cunningham DJ, Baumgartner RE, Federer AE, Richard MJ, Mithani SK. Elevated preoperative hemoglobin Alc associated with increased wound complications in diabetic patients undergoing primary, open carpal tunnel release. Plast Reconstr Surg. 2019;144(4):632e-638e.

13. Fang A, Hu SS, Endres N, Bradford DS. Risk factors for infection after spinal surgery. Spine (Phila Pa 1976). 2005;30(12):1460-1465.

14. Olsen MA, Nepple JJ, Riew KD, et al. Risk factors for surgical site infection following orthopaedic spinal operations. $J$ Bone Joint Surg Am. 2008;90(1):62-69.

15. von Elm E, Altman DG, Egger M, Pocock SJ, Gøtzsche PC, Vandenbroucke JP. The Strengthening the Reporting of Observational Studies in Epidemiology (STROBE) statement: guidelines for reporting observational studies. Lancet. 2007;370(9596):1453-1457.

16. Takahashi S, Suzuki A, Toyoda H, et al. Characteristics of diabetes associated with poor improvements in clinical outcomes after lumbar spine surgery. Spine (Phila Pa 1976). 2013;38(6):516-522.

17. Shin JI, Phan K, Kothari P, Kim JS, Guzman JZ, Cho SK. Impact of glycemic control on morbidity and mortality in adult idiopathic scoliosis patients undergoing spinal fusion. Clin Spine Surg. 2017;30(7):E974-E980.

18. Peng W, Liang Y, Lu T, et al. Multivariate analysis of incision infection after posterior lumbar surgery in diabetic patients: a single-center retrospective analysis. Medicine. 2019;98(23):e15935-e15935.

19. Shohat N, Tarabichi M, Tischler EH, Jabbour S, Parvizi J. Serum fructosamine. J Bone Joint Surg. 2017;99(22):19001907.

20. Ngaage LM, Osadebey EN, Tullie STE, et al. An update on measures of preoperative glycemic control. Plast Reconst Surgery Glob Open. 2019;7(5):e2240-e2240.

21. Piper K, DeAndrea-Lazarus I, Algattas H, et al. Risk factors associated with readmission and reoperation in patients undergoing spine surgery. World Neurosurg. 2018;110:e627e635.

22. Su AW, Habermann EB, Thomsen KM, Milbrandt TA, Nassr A, Larson AN. Risk factors for 30-day unplanned readmission and major perioperative complications after spine fusion surgery in adults: a review of the National Surgical Quality Improvement Program database. Spine (Phila $\mathrm{Pa}$ 1976). 2016;41(19):1523-1534.

23. Kim BD, Smith TR, Lim S, Cybulski GR, Kim JY. Predictors of unplanned readmission in patients undergoing lumbar decompression: multi-institutional analysis of 7016 patients. J Neurosurg Spine. 2014;20(6):606-616.

24. Modhia U, Takemoto S, Braid-Forbes MJ, Weber M, Berven SH. Readmission rates after decompression surgery in patients with lumbar spinal stenosis among Medicare beneficiaries. Spine (Phila Pa 1976). 2013;38(7):591-596.

25. Grauer JN, Leopold SS. Editorial: large database studies - what they can do, what they cannot do, and which ones we will publish. Clin Orthop Relat Res. 2015;473(5):15371539 . 
Disclosures and COI: The authors have no conflicts of interest, financial disclosures, or funding support.

Corresponding Author: Donald A. Ross, MD, Department of Neurological Surgery, Oregon Health \& Science University, 3303 SW Bond Ave,
CH8N, Portland, OR 87239. Phone: (503) 494-4314; Email: rossdo@ohsu. edu.

Published 19 August 2021

This manuscript is generously published free of charge by ISASS, the International Society for the Advancement of Spine Surgery. Copyright (C) 2021 ISASS. To see more or order reprints or permissions, see http://ijssurgery.com. 